\title{
The Consensus Hepatitis C Cascade of Care: standardized reporting to monitor progress toward elimination
}

Kelly Safreed-Harmon ${ }^{1}$; Sarah Blach ${ }^{2}$; Soo Aleman ${ }^{3,4}$; Knut Boe Kielland ${ }^{5}$; Signe Bollerup ${ }^{6}$; Graham Cooke $^{7}$; Olav Dalgard ${ }^{8,9}$; John F Dillon ${ }^{10}$; Gregory J Dore ${ }^{11}$; Ann-Sofi Duberg ${ }^{12}$; Jason Grebely ${ }^{11}$; Håvard Midgard $^{13}$; Kholoud Porter ${ }^{14}$; Homie Razavi ${ }^{2}$; Mark Tyndall ${ }^{15}$; Nina Weis ${ }^{6,16}$; Jeffrey V Lazarus ${ }^{1,17}$

1. Barcelona Institute for Global Health (ISGlobal), Hospital Clínic, University of Barcelona, Barcelona, Spain

2. Center for Disease Analysis Foundation, Lafayette, Colorado, United States

3. Department of Infectious Diseases, Karolinska University Hospital, Stockholm, Sweden

4. Department of Medicine Huddinge, Karolinska Institutet, Sweden

5. Norwegian National Advisory Unit on Concurrent Substance Abuse and Mental Health Disorders, Innlandet Hospital Trust, Brumunddal, Norway

6. Department of Infectious Diseases, Copenhagen University Hospital, Hvidovre, Copenhagen, Denmark

7. Faculty of Medicine, Imperial College London, United Kingdom

8. Department of Infectious Diseases, Akershus University Hospital, Lørenskog, Norway

9. Institute of clinical medicine, University of Oslo, Oslo, Norway

10. Division of Molecular and Clinical Medicine, School of Medicine, University of Dundee, Dundee, United Kingdom

11. The Kirby Institute, UNSW Sydney, Sydney, Australia

12. Department of Infectious Diseases, School of Medical Sciences, Örebro University, Örebro, Sweden

13. Department of Gastroenterology, Oslo University Hospital, Oslo, Norway

14. Institute for Global Health, University College London, London, United Kingdom

15. School of Population and Public Health, University of British Columbia, Vancouver, BC, Canada

16. Department of Clinical Medicine, Faculty of Health and Medical Sciences, University of Copenhagen, Copenhagen, Denmark

17. CHIP, WHO Collaborating Centre on HIV and Viral Hepatitis, Rigshospitalet, University of Copenhagen, Copenhagen, Denmark

\section{Corresponding author}

Jeffrey V Lazarus

Barcelona Institute for Global Health (ISGlobal), Hospital Clínic, University of Barcelona, Calle del Roselló 132, $4^{\text {th }}$ floor. ES-08036 Barcelona, Spain

Tel: (+34) 608703573

Jeffrey.Lazarus@isglobal.org

Summary: The Consensus Hepatitis C Cascade of Care is proposed for standardized reporting on progress toward key hepatitis $\mathrm{C}$ treatment targets. It provides detailed definitions for four essential cascade stages: infected, diagnosed, treated and cured. 


\begin{abstract}
Cascade-of-care $(\mathrm{CoC})$ monitoring is an important component of the response to the global hepatitis $\mathrm{C}$ virus (HCV) epidemic. CoC metrics can be used to communicate in simple terms the extent to which national and subnational governments are advancing on key targets, and $\mathrm{CoC}$ findings can inform strategic decision-making regarding how to maximize the progression of HCV-infected individuals to diagnosis, treatment and cure. The value of reporting would be enhanced if reporting entities utilized a standardized approach for generating their CoCs. We have described the Consensus HCV CoC that we developed to address this need and have presented findings from Denmark, Norway and Sweden, where it was piloted. We encourage the uptake of the Consensus HCV CoC as a global instrument for facilitating clear and consistent reporting via the World Health Organization (WHO) viral hepatitis monitoring platform and ensuring the accurate monitoring of progress toward WHO's 2030 hepatitis C elimination targets.
\end{abstract}

Keywords: Hepatitis C virus, cascade of care, disease elimination, monitoring, policy 
The first Global Health Sector Strategy on Viral Hepatitis, adopted by the World Health Organization (WHO) in 2016, calls for the elimination of viral hepatitis as a major public health threat by 2030. Specific 2030 targets for hepatitis C virus (HCV) include an $80 \%$ reduction in new chronic cases, a diagnosis level of $90 \%$, a treatment coverage level of $80 \%$, and a $65 \%$ reduction in deaths [1]. Meeting these targets will require many countries to greatly intensify their HCV control efforts. Only $20 \%$ of an estimated 71 million people with HCV were estimated to have been diagnosed by 2015 , with $7 \%$ of those diagnosed beginning treatment that year [2,3]. As of the end of 2017, 12 countries accounting for less than $15 \%$ of all HCV infections worldwide were reported to be on track to achieve WHO's 2030 HCV elimination goal $[3,4]$.

To support effective monitoring of global progress toward the viral hepatitis elimination targets, WHO has established a monitoring framework with ten core indicators addressing disease prevention, service coverage, incidence and mortality [5]. In 2018, WHO introduced the web-based Global Reporting System for Hepatitis (GRSH) to facilitate country reporting on these indicators. Four of the ten core indicators incorporated into GRSH are intended to be used for cascade of care (CoC) reporting [6].

The function of a $\mathrm{CoC}$ is to depict how many members of a population have progressed through each stage in a sequence of stages required for effective disease control, e.g., "diagnosis", "treatment" and "cure". In the HIV field, stakeholders have used $\mathrm{CoC}$ analyses to pinpoint where intensified efforts are needed to reduce fall-off along the cascade stages, e.g., a high level of HIV diagnosis but a low level of treatment may indicate a need to improve services for linking HIV-diagnosed people to care and retaining them in care [7]. The $\mathrm{CoC}$ also serves as the conceptual basis for the well-known global HIV control target of "90-90-90" introduced by UNAIDS in 2014: it calls for $90 \%$ of all people living with HIV to be diagnosed, for $90 \%$ of those diagnosed to be receiving treatment, and for $90 \%$ of those receiving treatment to be virally suppressed, all by 2020 [8].

In the HCV field, the introduction of interferon-free direct-acting antiviral (DAA) treatments with high cure rates in 2013 has been accompanied by a surge of interest in quantifying the HCV CoC. In settings worldwide, $\mathrm{HCV} \mathrm{CoC}$ findings are helping health system decision makers determine where the greatest gaps in testing, linkage to care and treatment are encountered among people with chronic HCV infection. HCV CoC reporting also indicates whether countries and the global community overall are on track to meet some of the key 2030 WHO service-level targets. However, a unified approach to defining the HCV CoC stages is lacking. Consequently, findings are being reported in highly diverse ways [9-14]. A similar situation in the HIV field limits the comparative value of HIV CoCs and makes it difficult to aggregate data for accurate assessments of progress at the regional and global levels [15]. Noting this problem, we sought to facilitate the standardized collection and reporting of HCV CoC data across settings by establishing a "consensus" HCV CoC. This article describes the new instrument, which can be used to strengthen the CoC component of WHO's GRSH reporting.

\section{Development and piloting of the Consensus Hepatitis C Cascade of Care}

The Consensus HCV CoC was developed by a study group comprising clinical, epidemiologic and public health experts from Europe, Australia, and North America. The process began with a review of published HCV CoCs (Box 1) as well as a review of methodological issues in HIV CoC monitoring [1519]. In a working paper based on this research, study group members considered which stages should be included in the Consensus HCV CoC, and how those stages should be defined. The task was restricted to the measurement of treatment-related milestones rather than a broader range of outcomes such as prevention outcomes. The working paper included a "zero draft" Consensus HCV CoC comprised of seven stages (Figure 1a). 
The study group discussed how to formulate the Consensus HCV CoC via teleconference and multiple rounds of e-mails between January and July 2018. In cases of disagreement, the issue was discussed until consensus was reached. All study group members agreed that essential stages should be similar to the four stages used by WHO in its most recently published global HCV cascade (Figure 1b), but that different terminology would be suitable for some or all of the stages. After considering the merits of various terms, the study group agreed to refer to the cascade stages as "infected", "diagnosed", "treated", and "cured".

There was extensive debate about whether the Consensus HCV CoC should include a stage for linkage to care following diagnosis. Some study group members emphasised that, in some settings, failure to effectively link HCV-diagnosed people to care has resulted in large numbers of people not progressing to treatment initiation $[20,21]$. The primary arguments against including a linkage-tocare stage were that developing an effective universal definition of linkage to care for reporting purposes would be very difficult and that very few national reporting systems would have data available for reporting accurately on such an item. While recognising that insufficient linkage to care is a major challenge in HCV elimination efforts, and that greater understanding of this stage is needed, the study group determined that its primary objective was to establish a clearly defined cascade of essential stages that should be regarded as immediate monitoring priorities for all countries. Furthermore, it was suggested that the advantages and disadvantages of collecting data to report on additional stages such as linkage to care may be weighed differently in different countries, and that country-level stakeholders should be the ones to decide which cascade stages in addition to the four essential ones are important to monitor in the context of the country's HCV epidemic and health system resources. A discussion about whether to include a stage after cure for accessing ongoing care ended with a similar conclusion: while the study group recognized it as an important stage for which more information is needed to guide programmatic activities, it was thought to be impractical to call on all countries to report on post-cure $\mathrm{HCV}$ care as part of their current CoC monitoring.

Definitions of the four cascade stages were drafted and the study group used published data and projections to consider how different definitions for some stages would result in different cascade outcomes (Figure 2). Study group members noted that definitions needed to be easily understandable to a broad range of stakeholders and practical for reporting entities to utilize while also taking into account methodological issues such as spontaneous clearance of HCV and the inevitable time interval between treatment initiation and sustained virologic response. The definitions agreed on and presented here provide the best balance among these competing concerns and therefore were selected for use in the pilot reporting exercise.

We sought to pilot the $\mathrm{CoC}$ in a region where the relevant health information was expected to be accessible. Leading HCV monitoring experts in Denmark, Finland, Iceland, Norway and Sweden, were invited to participate in the pilot study. Those from Denmark, Finland, Norway and Sweden agreed, but ultimately Finland was unable to provide the required data. Pilot participants were asked to use a Microsoft Excel template to report $\mathrm{CoC}$ data in accordance with our agreed definitions for the calendar years 2015-2017 (Appendix 1). Pilot participants were also asked to describe the methodologies used for generating the data. Data collection took place from August to November 2018.

We performed a descriptive analysis of all CoC findings and visually depicted the 2017 data in bar graphs along with data on HCV prevalence and HCV treatment for the years 2015-2017 (Box 2 and Figure 3 ). We then reviewed and refined the definitions of the $\mathrm{CoC}$ stages. The pilot definitions and final definitions are presented in Box 3.

\section{Using the Consensus Hepatitis C Cascade of Care to enhance global reporting}


We propose the Consensus HCV CoC not as an alternative to the WHO monitoring framework and GRSH platform, but rather as an instrument for clarifying what is being measured when reporting entities generate cascades that depict the numbers and proportions of HCV-infected individuals who have progressed to diagnosis, treatment and cure. The Consensus HCV CoC also provides researchers with a standardised measuring tool for cascade studies that are carried out apart from national monitoring activities. There are a multiplicity of ways to define the HCV cascade stages, and the current lack of clarity about definitions is problematic. The cascade that we have developed is synchronistic with WHO's guidance for CoC reporting, while addressing details of reporting in ways that promote greater clarity about what the data represent. Hence, we believe that carrying out national reporting in accordance with the Consensus HCV CoC conventions and then using the resulting data for GRSH reporting will improve the global community's understanding of how countries and regions are performing in relation to key HCV targets.

An integral component of Consensus HCV CoC reporting is documentation of how data were obtained for the four $\mathrm{CoC}$ stages, including information on assumptions on which estimates are based. In the case of the data reported in this study, the information is in an appendix to this article (Appendix 2). We propose that future HCV CoC reporting likewise include details of methodologies for calculating $\mathrm{CoC}$ data, including reporting that is disseminated through non-peer-reviewed channels such as government websites and reports. The objective should be to provide enough information to enable audiences to consider the robustness of the data critically.

A strength of the Consensus HCV CoC is that our definitions take into account the temporal dimension of $\mathrm{HCV} \mathrm{CoC}$ reporting. It is inherently challenging to formulate an HCV CoC that accurately expresses what is happening in reality because of the contradictory requirements of representing a snapshot of a specific point in time for some stages while also representing events that take place throughout the year of interest. Any approach to reporting both types of information in a single CoC will have drawbacks, and thus the most useful $\mathrm{CoC}$ reporting will be that which includes details of how the $\mathrm{CoC}$ stages were defined, as the notes accompanying Figure 3 demonstrate.

We also sought to provide context for interpreting $\mathrm{CoC}$ findings by incorporating data about trends over time into the reporting. While a bar graph showing the four CoC stages for 2017 is the most prominent visual element in Figure 3, the right side of the figure includes three smaller graphs: "Infected, 2015-2030", "Treated, 2015-2030" and "Cumulative treated, 2015-2030". The start date was selected because this was the first year that DAA treatment came into widespread use. The end date was selected to correspond to the WHO timeline for achieving HCV elimination targets. Including these graphs enables stakeholders to easily put the $\mathrm{CoC}$ findings for the most recent reporting period into historical context, and using consistent $\mathrm{CoC}$ definitions from one year to the next makes it easier to differentiate which changes are attributable to secular trends.

\section{Lessons learned from piloting the Consensus Hepatitis C Cascade of Care}

The Consensus HCV CoC definitions are straightforward and are practical for reporting entities to apply, as demonstrated by the fact that pilot participants were able to use the definitions to generate their cascades without any need for clarification about how definitions should be interpreted. Having a clearly defined Consensus HCV CoC, however, does not resolve underlying challenges associated with HCV monitoring, as illustrated by multiple aspects of the pilot experience.

First, when our pilot participants documented their methodologies for generating estimates for some cascade stages, they noted major data limitations (Appendix 2). Given the extensive health system resources in the three Nordic countries where piloting was conducted, the challenges encountered in generating estimates for some cascade stages are striking. We speculate that at least as much 
uncertainty may characterize findings from many other high-income countries, while cascades reported for low- and middle-income countries may reflect an even greater degree of uncertainty about some factors. Non-robust HCV prevalence estimates are a matter of particular concern since an accurate understanding of prevalence provides the foundation for health systems to implement effective disease control measures.

The pilot reporting also calls attention to multiple factors that may provide context for interpreting HCV CoC findings. The difference between the proportion of HCV-infected people diagnosed in three regions of Denmark (44\%) and the proportions diagnosed in Norway and Sweden (70\% and $80 \%$, respectively) is conspicuous in light of similarities in the health system characteristics and health system responses to HCV in the three countries. The Danish finding is somewhat consistent with findings from a study that used 2007 data from multiple registers to estimate a $54 \%$ national diagnosis level for Denmark [22]. It may be that different practices for reporting to surveillance registers in Denmark and the other two countries have resulted in the undercounting of diagnosed HCV cases in Denmark. Our inability to draw firm conclusions about this issue underscores the difficulty of accurately estimating the size of an undiagnosed HCV population even in settings with well-resourced health systems.

Another issue raised by the pilot reporting is the potential effect of treatment restrictions on the level of treatment uptake among people diagnosed with HCV. In Norway, for example, DAA-based treatment was available only to HCV patients with an F2 or higher fibrosis stage in 2015 and 2016, whereas patients infected with HCV genotypes 1 and 4 became eligible for DAA treatment regardless of their fibrosis stage in 2017, and with genotype 3 in March 2018. In Sweden, no stage F0-F1 patients, except for those with severe extrahepatic manifestations, were eligible for DAA treatment until restrictions were lifted at the beginning of 2018. This change triggered a sharp increase in the number of patients initiating treatment in the first months of 2018, and it was anticipated that the total number of people initiating treatment by the end of the year would be at least double that of the previous year. The presence or absence of treatment restrictions might potentially have a considerable impact on the degree of fall-off from "infected" to "diagnosed" to "treated" in the HCV $\mathrm{CoC}$, and it is thus advisable to consider the role of any such restrictions in interpreting and deciding how to act on $\mathrm{CoC}$ findings.

\section{Limitations and conclusions}

Although the Consensus HCV CoC is intended to be used in countries worldwide, we did not include representation from all regions, nor did we include investigators based in low- or middle-income countries. We sought to offset this limitation by drawing on our work experiences in Africa, Asia and Eastern Europe to consider how reporting capacity might be constrained in countries with weak health information systems. Nonetheless, some factors associated with the availability of data and the ways in which data are collected may become apparent only when the Consensus HCV CoC is used more widely, including in low-income countries.

The variable ability of health systems to report on sustained virological response (SVR) outcomes is an important issue to take into account when interpreting Consensus HCV CoC findings. In settings where considerable numbers of treated patients do not return for SVR assessment following the completion of treatment, reporting in accordance with the Consensus HCV CoC definitions may result in the underestimation of cured cases, since our fourth stage includes only treated patients with results indicating that SVR has been achieved. An alternate method of reporting on the number and proportion of treated people who are cured would be to simply estimate "cured" as an agreed-upon proportion of the number of people who initiated treatment, e.g. 95\%, or even to regard "treated" as a surrogate for "cured", since very high real-world SVR rates have been observed in patients who complete treatment [23]. As more evidence becomes available regarding this issue, it may become 
necessary to re-evaluate the strengths and weaknesses of these two different approaches to calculating the "cured" stage for reporting purposes.

In conclusion, we have proposed an $\mathrm{HCV} \mathrm{CoC}$ with clearly defined stages and have suggested how the findings should be communicated for optimal clarity. We encourage the uptake of the Consensus $\mathrm{HCV} \mathrm{COC}$ as a global instrument for facilitating clear and consistent reporting via the GRSH platform and ensuring the accurate monitoring of progress toward the WHO 2030 hepatitis C elimination targets. 


\section{NOTES:}

Author contributions. JVL and KSH conceived of the idea for the study and KSH managed the study. $J V L$ chaired the study group that made all methodological decisions, and GC, GD, HR, JFD, JG, KP, MT, NW, OD and SA served as study group members. S Blach contributed additional methodological input. ASD and SA reported cascade data for Sweden. HM, KBK and OD reported cascade data for Norway. NW and S Bollerup reported cascade data for Denmark. KSH drafted the manuscript. All authors reviewed multiple drafts and approved the final version for publication.

Acknowledgments. The authors thank Juan M Pericàs and Adrià Ramírez Mena for providing input on conceptual and methodological considerations in cascade-of-care reporting. The authors thank Hilde Kløvstad for assisting with the generation of data reported for Norway.

Funding. This study was supported by an unrestricted competitive Gilead Sciences Nordic Fellowship to CHIP, Rigshospitalet, University of Copenhagen, Denmark (FP_2016_10). JVL was funded by the Barcelona Institute for Global Health, Spain, via a Spanish Ministry of Science, Innovation and Universities Miguel Servet grant . GC is supported in part by the Biomedical Research Centre of Imperial College National Health Service Trust and a Research Professorship of the National Institute for Health Research.

Potential conflicts of interest. Outside of the scope of the submitted work: Soo Aleman has received research grants and speaker fees from AbbVie, Gilead Sciences, MSD and BMS. Sarah Blach is an employee of the Center for Disease Analysis (CDA) Foundation. CDA Foundation has received grants from the Centers for Disease Control and Prevention (CDC) Foundation, John C Martin Foundation, The Association of State and Territorial Health Officials (ASTHO), Zeshan Foundation, WHO Regional Office for the Western Pacific, WHO headquarters Geneva, Swiss Federal Office of Public Health, Brazil Ministry of Health, CDA, and private donors. CDA has received research funding from Gilead Sciences, AbbVie, and Intercept Pharma. Knut Boe Kielland has received speaker fees from MSD, Gilead and Abbvie. Signe Bollerup has no conflicts of interest to report. Graham Cooke has provided consultancy to Gilead Sciences and has received speaker fees from MSD. Olav Dalgard has received research grants and speaker fees from AbbVie, Gilead and MSD. John F Dillon has received institutional research grants and personal honoraria from Janssen, Roche, AbbVie, Gilead Sciences and MSD, and grants from Genedrive. Gregory J Dore has received research grants from BristolMyers Squibb, as well as research grants, honoraria, and non-financial support from AbbVie, Gilead Sciences, and MSD. Ann-Sofi Duberg has received speaker fees and/or served as an expert consultant for AbbVie, BMS, Gilead Sciences, Medivir and MSD. Jason Grebely has received research grants and honoraria from AbbVie, Cepheid, Gilead Sciences, and MSD, as well as non-financial support from Cepheid. Jeffrey $\mathbf{V}$ Lazarus has received research grants, speaker fees, and non-financial support from AbbVie, Gilead Sciences and MSD as well as speaker fees from Cepheid and Janssen. Havard Midgard has received speaker fees and advisory board fees from MSD, Gilead Sciences and Abbvie. Kholoud Porter has received honoraria from ViiV Healthcare and MSD. Homie Razavi reports grants from CDC Foundation, the Center for Disease Analysis, John Martin Foundation, ASTHO, Zeshan Foundation, and other private donors. In addition, his organization has received funding from Gilead Sciences, AbbVie, Intercept Pharma, and the Vaccine Impact Modeling Consortium. He is the managing director of CDA and the CDA Foundation. Kelly Safreed-Harmon has no conflicts of interest to report. Nina Weis has been a clinical investigator for Abbvie, BMS and MSD, and has been a speaker and participated in advisory boards for Abbvie, BMS, Gilead, GSK, and MSD. All fees were paid to her department. 
Figure 1a. The 'zero draft' Consensus Hepatitis C Cascade of Care

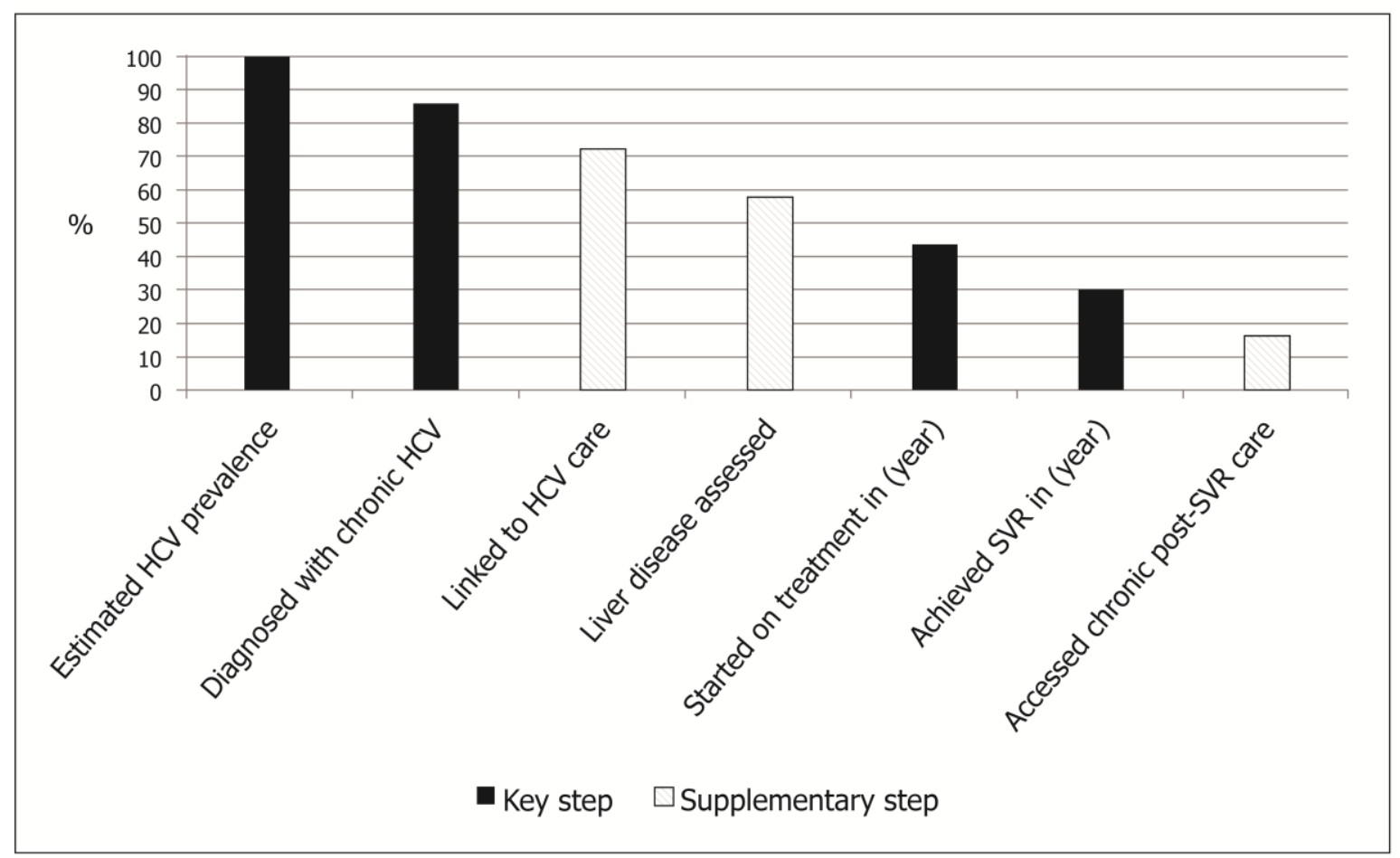

Figure 1b. Global estimates of progression across the HCV cascade of care from the World Health Organization's 2017 Global Hepatitis Report

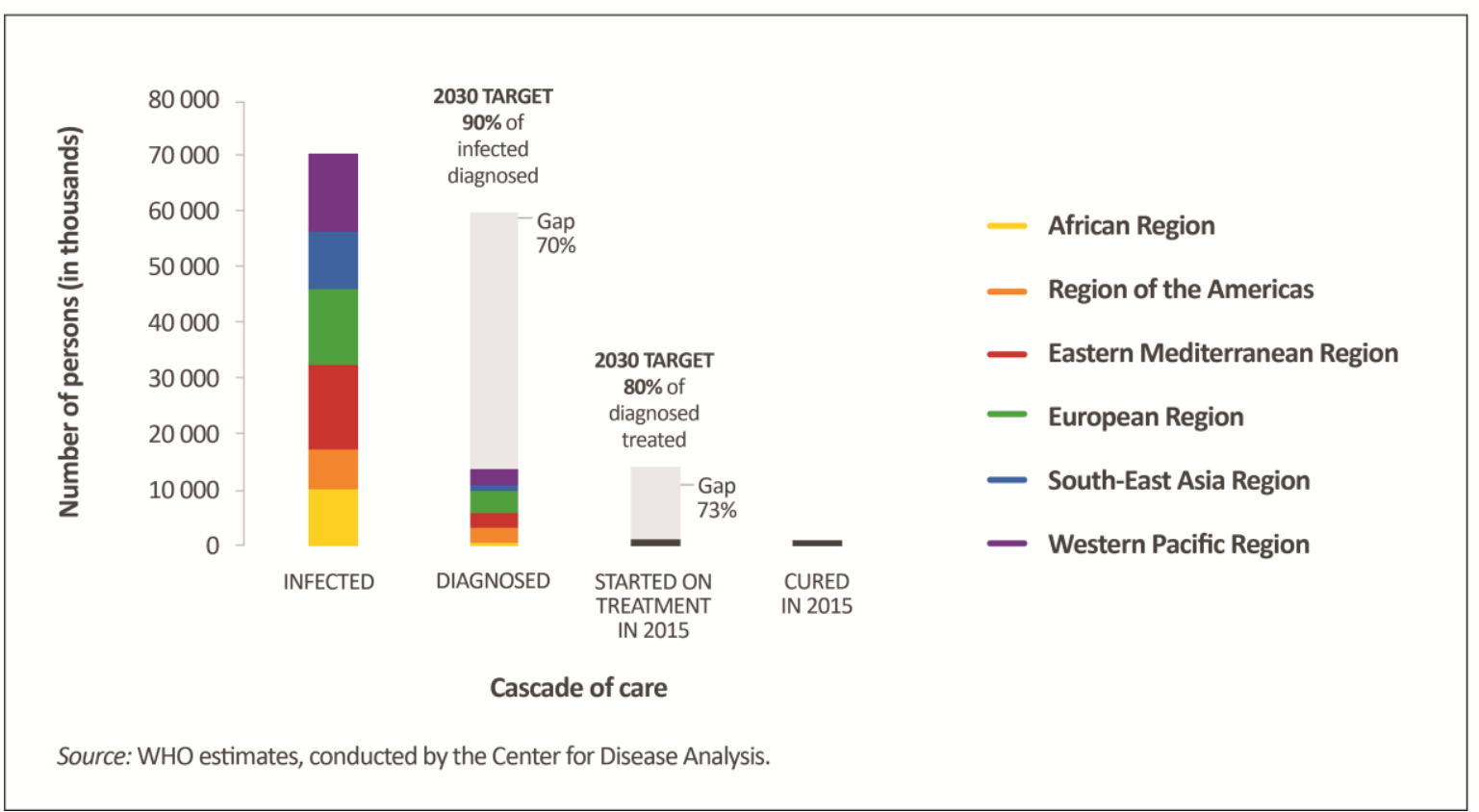


Figure 2. Variation in cascade outcomes resulting from changes in definitions for stages*

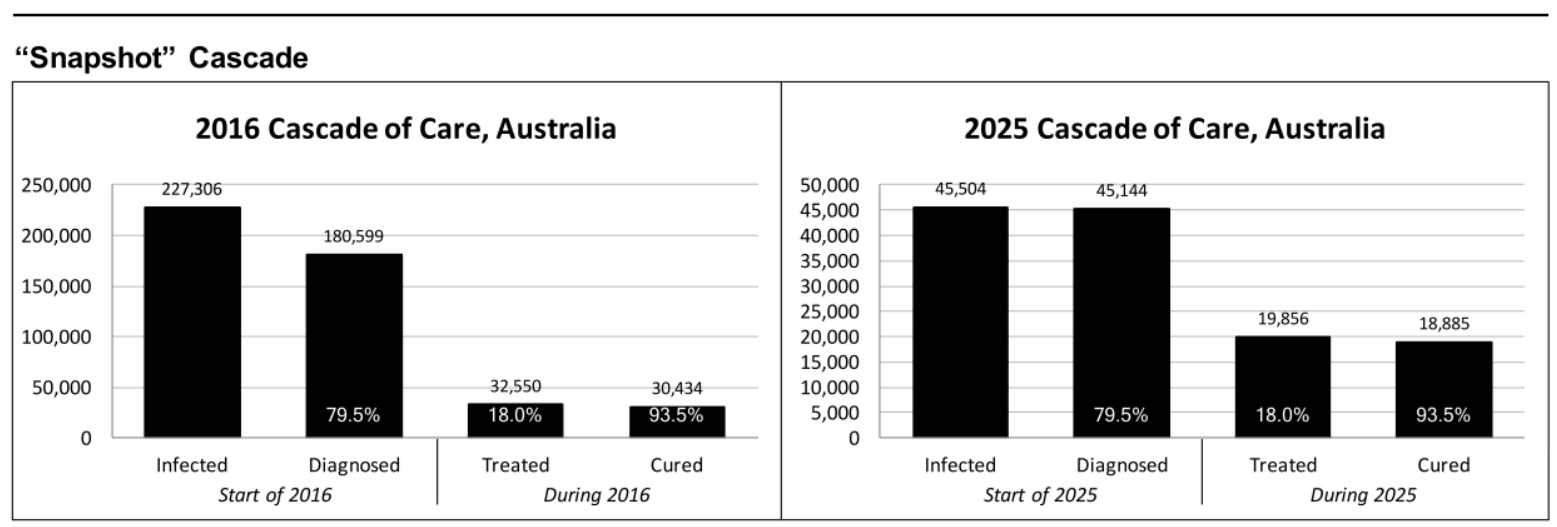

Infected $=$ had HCV infection on 1 January of the year of interest. Diagnosed $=$ diagnosed as of 1 January of the year of interest. Treated = initiated treatment at any time during the year of interest. Cured = cured at any time during the year of interest.

\section{"Cumulative" Cascade}

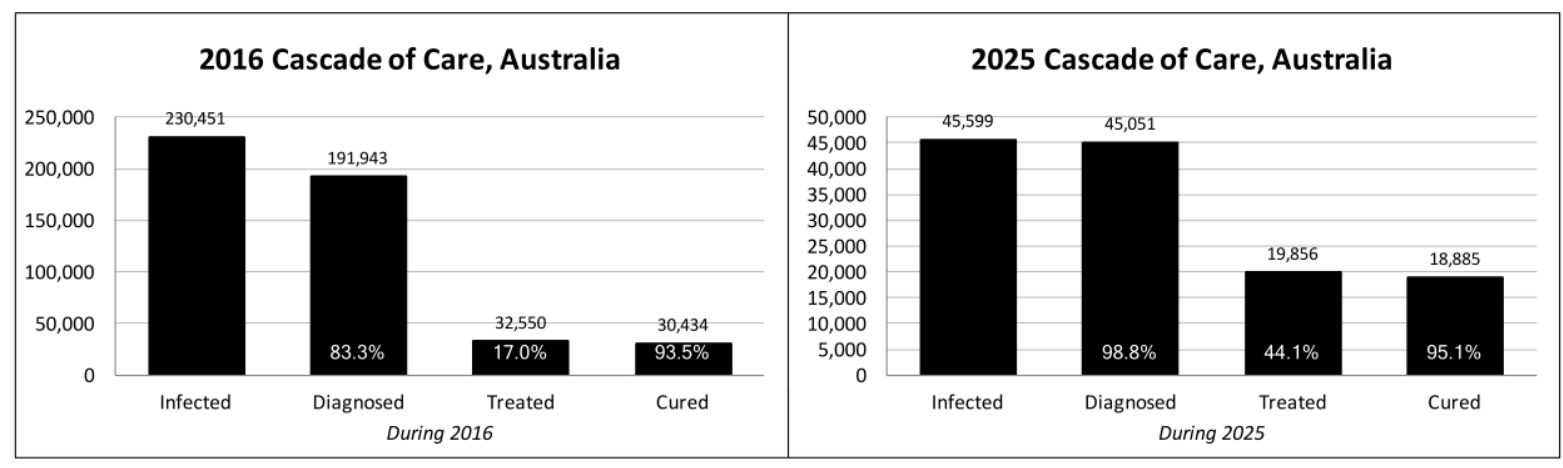

Infected = had HCV infection on any day during the year of interest. Diagnosed = diagnosed before or during the year of interest, e.g., had an HCV diagnosis on any day during 2016 (including still infected at beginning 2016 and still alive at end 2016; excluding cured before 2016).

Treated = initiated treatment at any time during the year of interest. Cured = cured at any time during the year of interest.

\section{"Hybrid" Cascade}

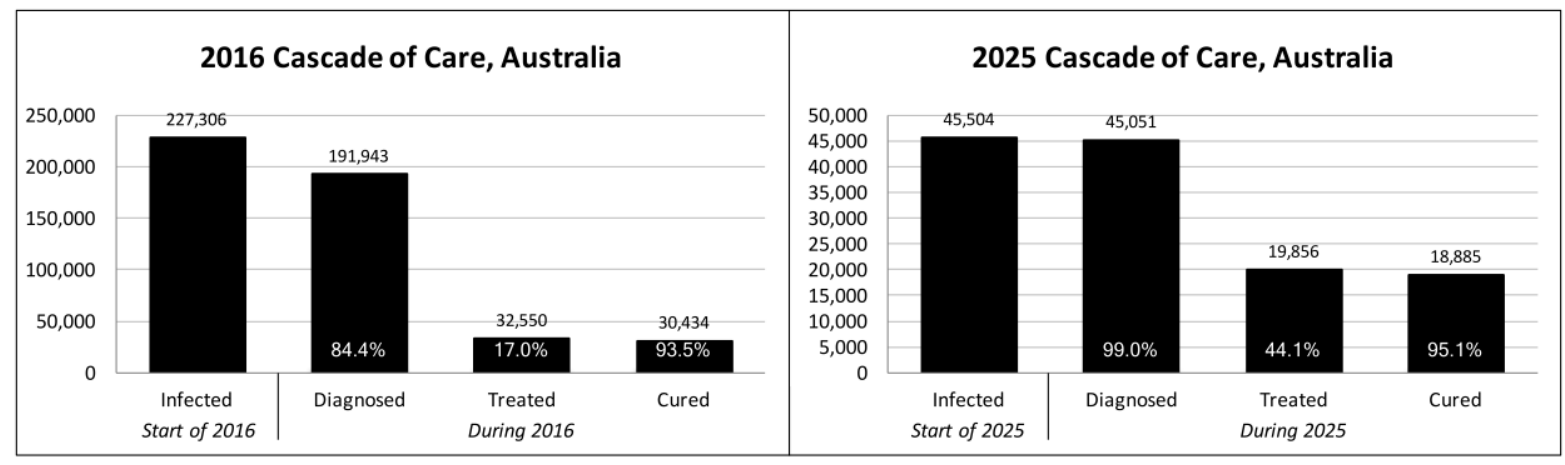

Infected = had HCV infection on 1 January of the year of interest. Diagnosed = diagnosed before or during the year of interest, e.g., had an HCV diagnosis on any day during 2016 (including still infected at beginning 2016 and still alive at end 2016; excluding cured before 2016). Treated $=$ initiated treatment at any time during the year of interest. Cured = cured at any time during the year of interest.

* All 2016 cascades were populated with data from HIV, Viral Hepatitis and Sexually Transmissible Infections in Australia: Annual Surveillance Report 2017 (https://kirby.unsw.edu.au/sites/default/files/kirby/report/SERP_Annual-Surveillance-Report-2017_compressed.pdf). All 2025 cascades were populated with unpublished data from the Polaris Observatory (https://cdafound.org/polaris/). 
Figure 3. 2017 Consensus HCV Cascades of Care, Denmark, Norway and Sweden

\section{Denmark (subnational)*}
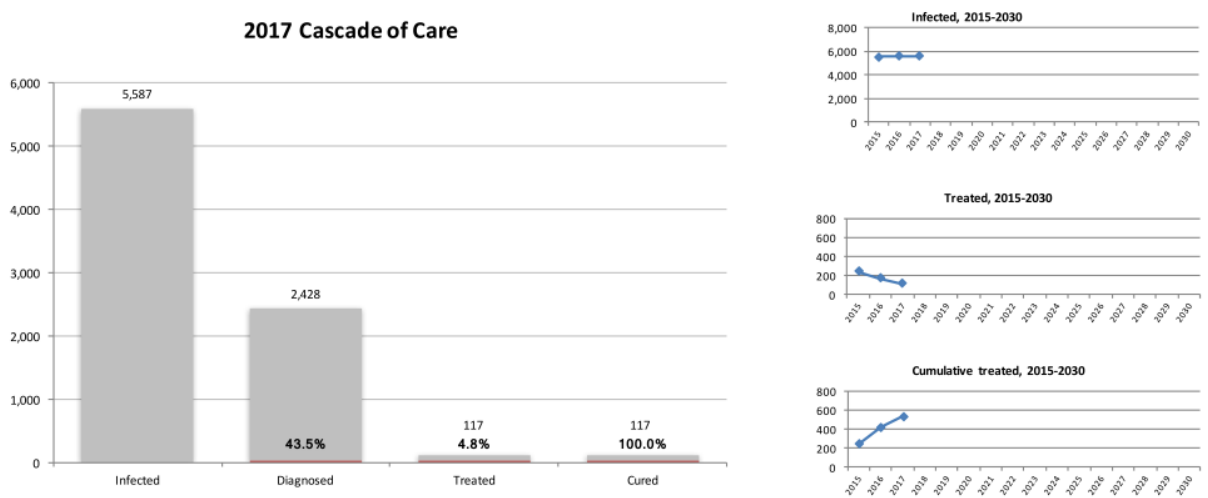

\section{Norway}
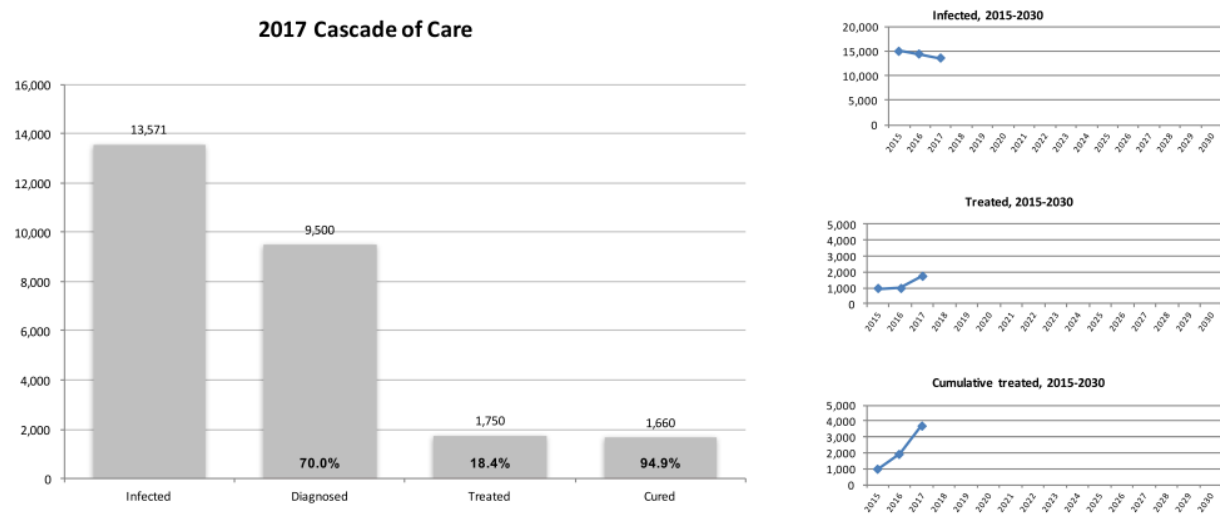

\section{Sweden}
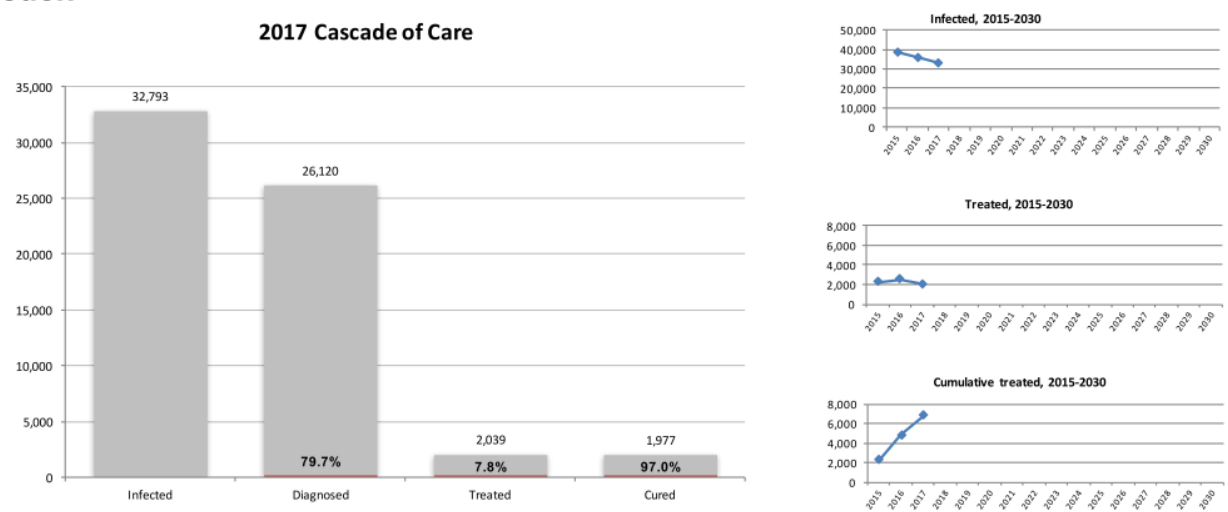

Infected $=$ Number of people estimated to have viremic HCV infection on 1 January 2017. Diagnosed = Number of people who received a diagnosis of viremic $\mathrm{HCV}$ infection before or during 2017, were still infected at the beginning of 2017 and were still alive at the end of 2017. This number excludes people who were cured of their HCV infection or who experienced spontaneous not be counted in this group.) Treated $=$ Number of people who initiated HCV treatment at any time during 2017 (all types of treatment, including interferon-based regimens). Cured $=$ Estimated number of people who attained a sustained virologic response (SVR) at any time during 2017. This estimate is determined by first calculating the percentage of people who attained SVR from among all people who initiated treatmentin 2017 and whose SVR outcome is known, including people whose SVR was not observed until 2018. This "actual SVR" percentage is then multiplied by the number of people who initiated HCV treatment at any time during 2017. The resulting number is the estimated number of people who attained SVR at any

The \% in diagnosed columns is calculated using number of people diagnosed as the numerator and number of people infected as the denominator. The \% in treated columns is calculated

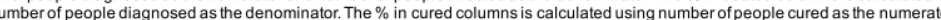

Dengerial Capital Region, Zealand and Northern Jutland. 


\section{References}

${ }^{1}$ World Health Organization. Global Health Sector Strategy on Viral Hepatitis, 2016-2021: towards
ending viral hepatitis. Geneva: World Health Organization; 2016. Available at:
http://apps.who.int/iris/bitstream/ 10665/246177/1/WHO-HIV-2016.06-eng.pdf. Accessed 26 April
2018.
${ }^{2}$ World Health Organization. Global hepatitis report 2017. Geneva: World Health Organization; 2017.
Available at: https://apps.who.int/iris/bitstream/handle/10665/255016/9789241565455-
eng.pdf;jsessionid=F36776F90FEEABBDFDEFFA1FF11B439F?sequence=1. Accessed 19 February 2019.
${ }^{3}$ Polaris Observatory HCV Collaborators. Global prevalence and genotype distribution of hepatitis C
virus infection in 2015: a modelling study. Lancet Gastroenterol Hepatol 2017; 2 (3):161-76.
${ }^{4}$ CDA Foundation. Just 12 countries worldwide on track to eliminate hepatitis C infection by 2030,
with United Kingdom, Italy and Spain among those joining the list [web page]. 2018. Available at:
http://cdafound.org/just-12-countries-worldwide-on-track-to-eliminate-hepatitis-c-infection-by-
2030-with-united-kingdom-italy-and-spain-among-those-joining-the-list/. Accessed 30 November
2018.
${ }^{5}$ World Health Organization. Monitoring and evaluation for viral hepatitis B and C: recommended
indicators and framework. Geneva: World Health Organization; 2016. Available at:
https://apps.who.int/iris/bitstream/handle/10665/204790/9789241510288 eng.pdf?sequence=1. Accessed 16 May 2019.

${ }^{6}$ World Health Organization. Global reporting system for hepatitis (GRSH)-project description. 2018. Available at: https://www.who.int/hepatitis/GRSH-Project description.pdf?ua=1. Accessed 16 May 2019.

${ }^{7}$ Chkhartishvili N, Chokoshvili O, Dvali N, Abutidze A, Sharvadze L, Tsertsvadze T. Significant improvements are needed in HIV care continuum to meet 90-90-90 targets in Georgia. J Int Assoc Provid AIDS Care 2016; 15(6):451-4.

${ }^{8}$ UNAIDS. 90-90-90: an ambitious treatment target to help end the AIDS epidemic. Geneva: UNAIDS; 2014. Available at http://www.unaids.org/sites/default/files/media asset/90-90-90 en.pdf. Accessed 16 May 2019.

${ }^{9}$ Ford MM, Johnson N, Desai P, Rude E, Laraque F. From care to cure: demonstrating a model of clinical patient navigation for hepatitis $\mathrm{C}$ care and treatment in high-need patients. Clin Infect Dis $2017 ; 64(5): 685-91$.

${ }^{10}$ Hochstatter KR, Hull SJ, Stockman LJ, Stephens LK, Olson-Streed HK, Ehlenbach WJ, et al. Using database linkages to monitor the continuum of care for hepatitis $C$ virus among syringe exchange clients: experience from a pilot intervention. Int J Drug Policy 2017; 42:22-5.

${ }^{11}$ Iversen J, Grebely J, Catlett B, Cunningham P, Dore GJ, Maher L. Estimating the cascade of hepatitis C testing, care and treatment among people who inject drugs in Australia. Int J Drug Policy 2017; 47:77-85.

12 Janjua NZ, Kuo M, Yu A, et al. The population level cascade of care for hepatitis C in British Columbia, Canada: The BC Hepatitis Testers Cohort (BC-HTC). EBioMedicine 2016; 12:189-95.

${ }^{13}$ Linas BP, Barter DM, Leff JA, et al. The hepatitis $C$ cascade of care: identifying priorities to improve clinical outcomes. PLoS One 2014; 9(5):e97317.

${ }^{14}$ Yehia BR, Schranz AJ, Umscheid CA, Lo Re V 3rd. The treatment cascade for chronic hepatitis C virus infection in the United States: a systematic review and meta-analysis. PLoS One 2014; 9(7):e101554.

${ }^{15}$ Gourlay AJ, Pharris AM, Noori T, et al. Towards standardized definitions for monitoring the continuum of HIV care in Europe. AIDS 2017; 31(15):2053-8.

${ }^{16}$ Drew RS, Rice B, Ruutel K, Delpech V, Attawell KA, Hales DK, et al. HIV continuum of care in Europe and Central Asia. HIV Med. 2017;18(7):490-9. 
${ }^{17}$ Granich R, Gupta S, Hall I, Aberle-Grasse J, Hader S, Mermin J. Status and methodology of publicly available national HIV care continua and 90-90-90 targets: A systematic review. PLoS Med. 2017;14(4):e1002253.

${ }^{18}$ Medland NA, McMahon JH, Chow EP, Elliott JH, Hoy JF, Fairley CK. The HIV care cascade: a systematic review of data sources, methodology and comparability. J Int AIDS Soc. 2015;18:20634.

${ }^{19}$ Perlman DC, Jordan AE, Nash D. Conceptualizing Care Continua: Lessons from HIV, Hepatitis C Virus, Tuberculosis and Implications for the Development of Improved Care and Prevention Continua. Front Public Health. 2016;4:296.

${ }^{20}$ Day E, Hellard M, Treloar C, Bruneau J, Martin NK, Øvrehus A, et al. Hepatitis C elimination among people who inject drugs: Challenges and recommendations for action within a health systems framework. Liver Int. 2019 Jan;39(1):20-30.

${ }^{21}$ Zuckerman A, Douglas A, Nwosu S, Choi L, Chastain C. Increasing success and evolving barriers in the hepatitis $C$ cascade of care during the direct acting antiviral era. PLoS ONE. 2018;13(6):e0199174. ${ }^{22}$ Christensen PB, Hay G, Jepsen P, et al. Hepatitis C prevalence in Denmark - an estimate based on multiple national registers. BMC Infect Dis 2012; 12:178.

${ }^{23}$ Ferreira VL, Leonart LP, Tonin FS, Borba HHL, Pontarolo R. Sustained Virological Response in Special Populations with Chronic Hepatitis C Using Interferon-Free Treatments: A Systematic Review and Meta-analysis of Observational Cohort Studies. Clin Drug Investig. 2018 May;38(5):389-400. 